UVX 2008 (2009) 7-14

(C) EDP Sciences, 2009

DOI: $10.1051 / \mathrm{uvx} / 2009003$

\title{
Dynamique temporelle des sources $X$ créées par laser sub-picoseconde
}

\author{
S. Bastiani-Ceccotti ${ }^{1}$, P. Renaudin ${ }^{2}$, F. Dorchies ${ }^{3}$, M. Harmand ${ }^{3}$, \\ O. Peyrusse ${ }^{3}$, S. Jacquemot ${ }^{1,2}$, E. Brambrink ${ }^{1}$, M. Geisse ${ }^{4}$ et P. Audebert ${ }^{1}$ \\ ${ }^{1}$ LULI, École Polytechnique, CNRS, CEA, UPMC, 91128 Palaiseau Cedex, France \\ ${ }^{2}$ CEA, DAM, DIF, 91297 Arpajon, France \\ ${ }^{3}$ Université de Bordeaux-CNRS-CEA, Centre Lasers Intenses et Applications (CELIA), \\ 33405 Talence, France \\ ${ }^{4}$ Sandia National Laboratories, PO Box 5800, Albuquerque, NM 87185-1193, USA
}

\begin{abstract}
Résumé. Nous présentons les résultats d'une expérience récente menée sur l'installation laser 100TW du LULI. Dans cette expérience, nous avons irradié des cibles solides de différents numéros atomiques avec des impulsions laser d'énergie jusqu'à $26 \mathrm{~J}$ et de durée allant de $300 \mathrm{fs}$ à 33 ps. Le diagnostic principal était une caméra à balayage de fente, couplée à un cristal de Bragg conique, qui a permis la mesure résolue en temps de l'émission $X$ du plasma créé dans la gamme 7,6-8,1 $\AA$. Nous avons pu suivre la modification spectaculaire des structures spectrales induite par la diminution de la durée de l'impulsion laser. Le spectre observé évolue d'une forme caractéristique d'une interaction en régime nanoseconde, avec des raies fines et bien résolues, à une forme de type "patatoïde », liée à la présence de structures spectrales extrêmement élargies, soit par effet Stark soit par simple accumulation de raies satellites. Les spectres mesurés montrent également une très forte dépendance en temps, ce qui permet de déterminer l'évolution des paramètres hydrodynamiques du plasma émissif et de les comparer aux simulations réalisées avec le code d'hydrodynamique radiative CHIVAS. Les résultats expérimentaux ont également été confrontés à des simulations faites à l'aide du code collisionnel-radiatif de cinétique atomique AVERROES/TRANSPEC.
\end{abstract}

\section{INTRODUCTION}

Le développement des lasers à dérive de fréquence, délivrant des impulsions courtes (sub-picosecondes) et intenses (au-delà de $10^{17} \mathrm{~W} / \mathrm{cm}^{2}$ ), a permis l'essor des recherches, tant expérimentales que théoriques, sur les propriétés, notamment radiatives, de la matière chaude et dense ( $T_{e}$ quelques centaines d'eV, $n_{e}$ jusqu'à quelques $10^{23} \mathrm{~cm}^{-3}$ ). Plusieurs campagnes d'expériences ont ainsi été réalisées depuis la fin des années 90 dans le domaine des rayons X. Visant, dans un premier temps, simplement à caractériser sur le plan spectral l'émission de ces plasmas à haute densité d'énergie [1-7], elles ont plus récemment porté sur la mesure de sa durée [8]. Cette étude des sources X se poursuit sur les installations du LULI ; elle se concentre actuellement sur l'analyse de l'influence de la durée de l'impulsion laser sur le spectre, en fonction du numéro atomique, afin de mieux comprendre la physique de l'interaction mise en jeu et de fournir des données pertinentes pour la validation des codes de physique atomique développés par ailleurs.

\section{DISPOSITIF EXPÉRIMENTAL}

L'expérience a été réalisée sur l'installation laser 100TW du LULI. Nous avons utilisé des impulsions laser d'énergie jusqu'à $26 \mathrm{~J}$, à une longueur d'onde de 1,06 $\mu \mathrm{m}$. La durée (à mi-hauteur) des impulsions a été variée de 300 fs à 33 ps, en gardant constante l'énergie laser. Le faisceau laser a été focalisé au moyen d'une parabole hors axe sur des cibles solides de différents matériaux : Al, Sm, purs ou recouverts d'une couche de vanadium (V), ou Se. $60 \%$ de l'énergie laser était alors contenue dans une tache focale elliptique d'environ $55 \mu \mathrm{m}^{2}$. L'émission $\mathrm{X}$ a été enregistrée au moyen d'une caméra à sténopé et de 


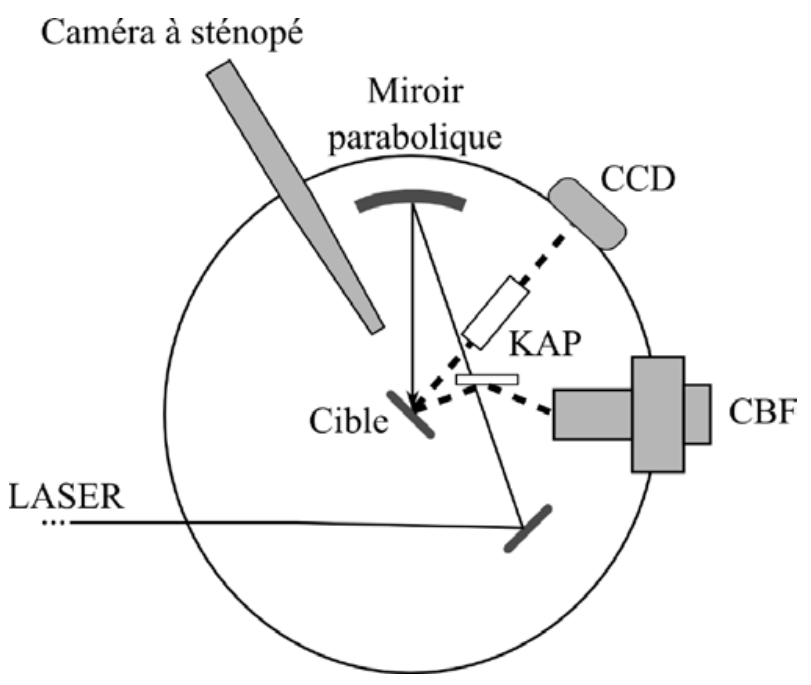

Figure 1. Schéma du montage expérimental et implantation des différents diagnostics.

deux spectromètres. La caméra à sténopé a été essentiellement utilisée pour contrôler la qualité de l'interaction du laser avec la cible. Chaque spectromètre était constitué d'un cristal conique [9] de KAP couplé soit à une caméra à balayage de fente $(\mathrm{CBF}) \mathrm{X}$ ultra-rapide [10] soit à une CCD X. La figure 1 montre un schéma du montage de l'expérience. Le spectromètre résolu en temps, diagnostic principal, a permis de recueillir des spectres avec une résolution spectrale de $5 \mathrm{~m} \AA$ et une résolution temporelle meilleure que 1 ps (voir figure 2). L'ensemble du dispositif a conduit à une gamme spectrale comprise entre 7,6 et 8,1 $\mathrm{\AA}$. Aprés traitement (incluant une correction de la courbure du spectre induite par la $\mathrm{CBF}$ ), les images sont analysées selon deux axes de coupe pour obtenir soit des spectres à un instant donné soit l'évolution temporelle d'une raie à une longueur d'onde donnée.

L'impulsion délivrée à 1,06 $\mu \mathrm{m}$ par l'installation 100TW présente un important piédestal lié à l'ASE (environ un millième de l'énergie laser totale sur $500 \mathrm{ps})$. Le contraste dégradé qu'il induit $\left(\sim 10^{-6}\right.$ en intensité) n'est pas suffisant à garantir qu'il n'y ait pas de création d'un pré-plasma lors de l'interaction. La couche de V recouvrant une partie des cibles avait pour objectif d'absorber l'énergie contenue dans le piédestal, laissant ainsi l'impulsion sub-ps interagir avec la cible (encore) solide.

\section{ANALYSE DES RÉSULTATS EXPÉRIMENTAUX}

\subsection{Emission de couche $\mathrm{K}$ : cibles en $\mathrm{Al}$ et en V/Al}

Les cibles «massives» étaient des feuilles d'Al de $50 \mu \mathrm{m}$ d'épaisseur, les cibles bi-couches étaient constituées d'une couche de $0,2 \mu \mathrm{m}$ de V déposée sur une feuille de $20 \mu \mathrm{m}$ d'Al. Sur la figure 2 nous avons reporté les images enregistrées lors de l'irradiation de cibles en V/Al et en $\mathrm{Al}$ pur par une impulsion laser de durée $300 \mathrm{fs}$ et d'intensité de l'ordre de $4,5 \pm 0,3 \cdot 10^{19} \mathrm{~W} / \mathrm{cm}^{2}$. Des différences très nettes peuvent être relevées. Outre un différentiel d'intensité (les deux images ont la même échelle de gris), on observe un fort élargissement des structures spectrales, jusqu'à en devenir à peine identifiables dans le cas de la cible bi-couche. Ce comportement est également visible sur la figure 3 qui montre les spectres enregistrés pour les deux types de cibles et différentes conditions laser, mais à des temps identiques pour chaque durée laser.

Dans le cas des cibles en V/Al, l'influence de la durée d'impulsion sur la forme du spectre émis est évidente. Pour une impulsion laser de 3 ps de durée, la raie de résonnance (He $\alpha$ à 7,757 ̊) et les satellites lithiumoïdes sont bien définis, alors que pour l'impulsion de $300 \mathrm{fs}$ de durée ces structures 

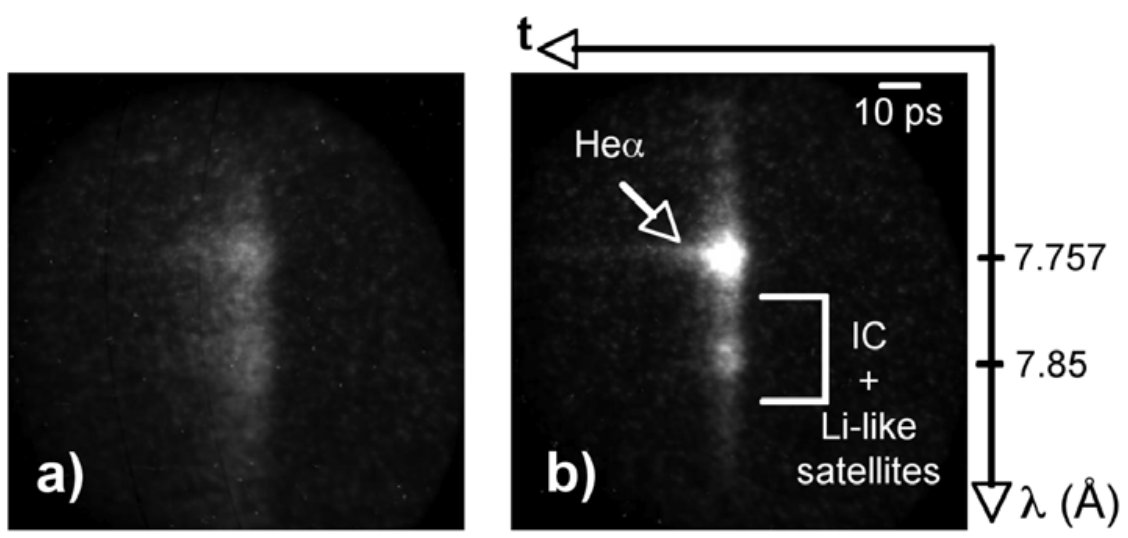

Figure 2. Images enregistrées par la caméra à balayage de fente lors de l'irradiation avec une impulsion laser de $300 \mathrm{fs}$ de durée d'une cible en a) V/Al (intensité de $4.3 \cdot 10^{19} \mathrm{~W} / \mathrm{cm}^{2}$ ) et b) Al pur (intensité de $4.8 \cdot 10^{19} \mathrm{~W} / \mathrm{cm}^{2}$ ). La notation des raie est: $\mathrm{He} \alpha: 1 s^{2}-1 s 2{ }^{1} \mathrm{P}_{1}$; IC: $1 s^{2}-1 s 2 p{ }^{3} \mathrm{P}_{1}$; satellites lithiumoïdes (Li-like) : $1 s^{2} n l-1 s 2 p n l$.

spectrales sont à peine reconnaissables tant elles sont élargies. On peut toutefois constater, dans les deux cas, l'absence, totale ou presque, de la raie d'intercombinaison (à 7,806 ̊). Cela indique que le plasma créé est très dense, au-delà de $10^{22} \mathrm{~cm}^{-3}$, et ce d'autant plus que l'impulsion laser est brève. De plus, dans le cas de l'impulsion laser de $3 \mathrm{ps,} \mathrm{on} \mathrm{peut} \mathrm{remarquer} \mathrm{que} \mathrm{le} \mathrm{rapport} \mathrm{des} \mathrm{satellites}$ lithiumoïdes $\{j, k, l\} /\{a-d, q, r\}$ varie fortement au cours du temps, ce qui suggère une cinétique atomique fortement non stationnaire. A contrario, les spectres émis par les cibles en Al pur sont analogues à ceux obtenus depuis longtemps en régime ns : le rapport d'intensité entre la raie de résonance $\mathrm{He} \alpha$ et les satellites lithiumoïdes indique que le plasma émissif est chaud, et la présence de la raie d'intercombinaison indique que le plasma émissif est peu dense. On peut donc en conclure que la couche de $\mathrm{V}$ a pleinement joué son rôle d'absorbant ; l'interaction laser-aluminium s'est produite, dans le cas des cibles V/Al, à une densité proche de celle du solide, alors que, dans les cibles en Al pur, elle s'est produite dans un plasma pré-formé sous-dense. Enfin, il est important de souligner que l'émission des cibles en $\mathrm{Al}$ pur est bien plus intense que celle des cibles en V/Al. Ceci sera discuté dans la section 4.

Le comportement temporel des différentes raies spectrales est présenté sur la figure 4. Pour chaque type de cible nous avons tracé les données obtenues pour trois durées de l'impulsion laser. L'axe horizontal (temporel) n'a pas de signification absolue, les courbes ayant été translatées pour en faciliter la comparaison. Dans le cas des cibles en Al pur, l'émission de la raie He $\alpha$ dure plus longtemps que celles des satellites lithiumoïdes, ces derniers n'étant plus émis pendant la phase de recombinaison du plasma. De plus, l'émission plus intense de la raie de résonnance en l'absence de la couche de V (les unités arbitraires de l'axe vertical étant identiques pour tous les graphes) indique que, sans cette dernière, le plasma subit une expansion hydrodynamique plus rapide (cf. section 4). Le vanadium joue donc à la fois un rôle d'absorbant (comme montré au paragraphe précédent) mais également de tampon, permettant ainsi au plasma de rester dense.

\subsection{Emission de couche $L$ : cibles en $\mathrm{Se}$}

La figure 5 montre les spectres obtenus avec des cibles de Se, de 240 Å d'épaisseur, pour trois durées de l'impulsion laser, et à un temps correspondant au maximum d'émission. Les structures $2 p-3 d$ néonoïdes, sodiumoïdes et fluorö̈des sont clairement identifiables, et elles sont notées 3C et 3D sur la figure. Comme dans le cas de l'Al, le spectre obtenu avec l'impulsion laser de plus courte durée présente des structures moins intenses, comme «gommées »: la raie 3C est pratiquement absente et la raie 3D est 

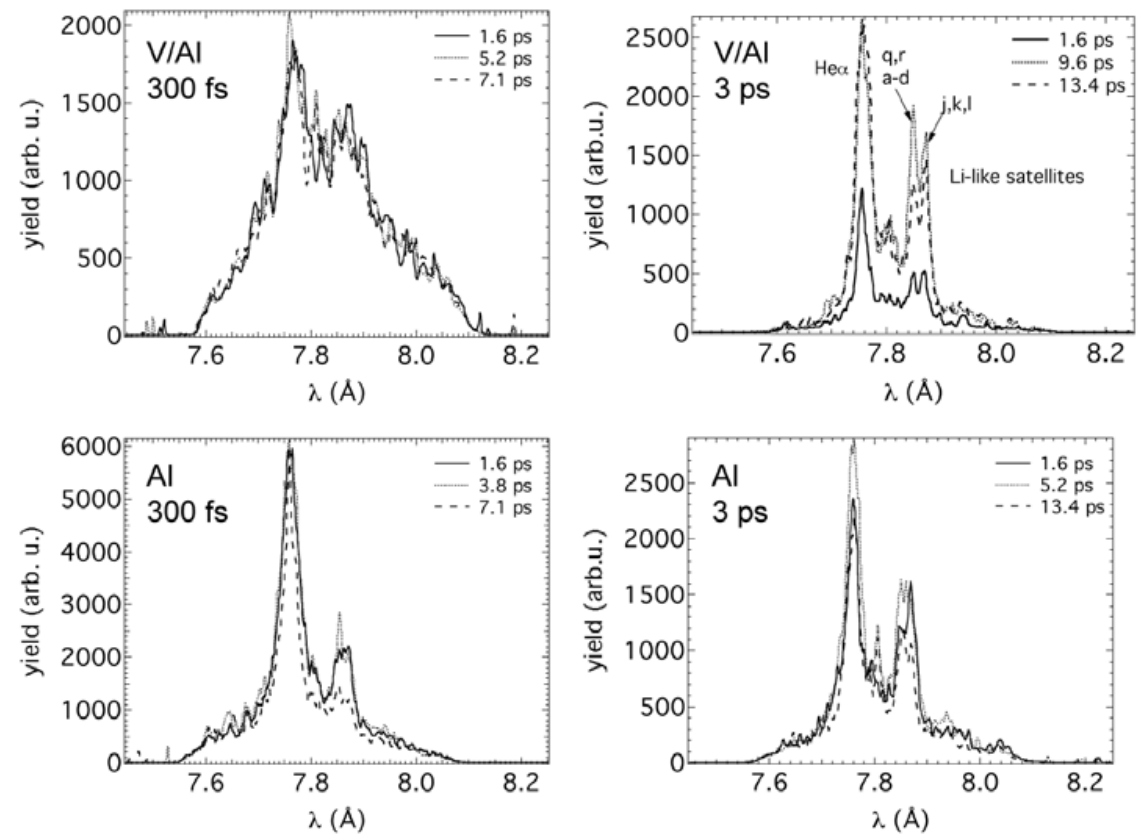

Figure 3. Spectres d'Al obtenus avec des cibles soit en Al pur soit en V/Al. La durée de l'impulsion laser et son intensité sont : a) $300 \mathrm{fs}, 4,3 \cdot 10^{19} \mathrm{~W} / \mathrm{cm}^{2}$; b) $3 \mathrm{ps}, 2,3 \cdot 10^{18} \mathrm{~W} / \mathrm{cm}^{2}$; c) $300 \mathrm{fs}, 4,8 \cdot 10^{19} \mathrm{~W} / \mathrm{cm}^{2}$; d) $3 \mathrm{ps}$, $2,7 \cdot 10^{18} \mathrm{~W} / \mathrm{cm}^{2}$.
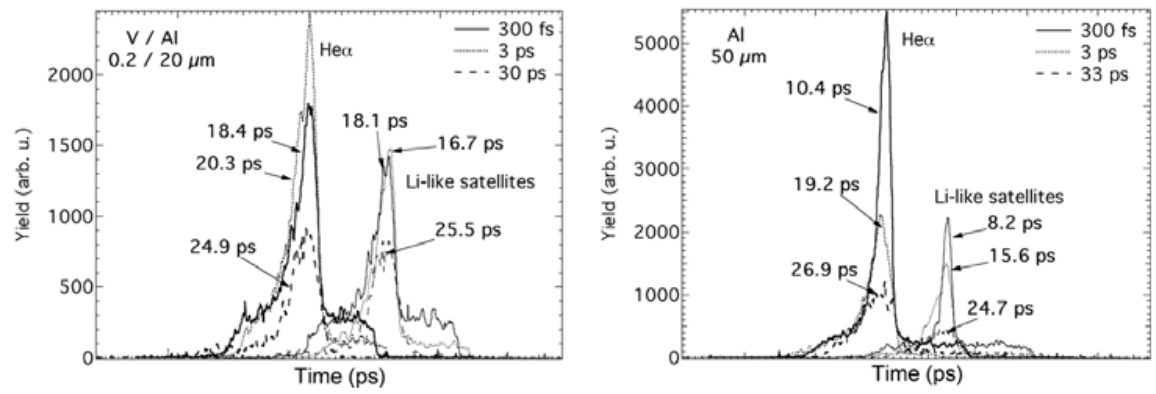

Figure 4. Durées d'émission de la raie He $\alpha$ et des satellites lithiumoïdes pour deux types de cibles, et pour trois durées laser.

très faible. Des calculs antérieurs [15] ont montré que le rapport d'intensité des satellites sodiumoïdes et néonoïdes est principalement sensible à la temperature électronique (figure 6). Nous avons donc calculé le rapport d'intensité des raies $\{2 p-3 d\} /\{3 C+3 D\}$ à partir des intensités mesurées, rapport qui varie de 0,41 pour l'impulsion laser de 300 fs, à 0,26 pour celle de 33 ps. Nous pouvons donc borner les valeurs des paramètres hydrodynamiques du plasma, entre 400 et $500 \mathrm{eV}$ pour la température électronique et de $10^{20}$ jusqu'á $2 \cdot 10^{21} \mathrm{~cm}^{-3}$ pour la densité électronique (région grisée sur la figure 6 ). Cette conclusion sera reprise dans la section 4.

\subsection{Emission de couche $M$ : cibles en $\mathrm{Sm}$ et en V/Sm}

En ce qui concerne l'émission de couche M, la figure 7 montre les spectres obtenus au pic de l'émission pour trois conditions d'irradiation laser. Les transitions mesurées correspondent aux transitions $3 d-4 f$ 


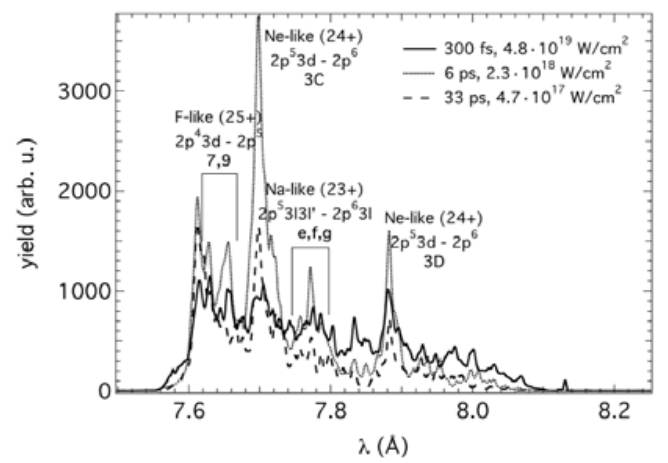

Figure 5. Spectres du Se au pic de l'émission, pour trois différentes durées de l'impulsion laser.

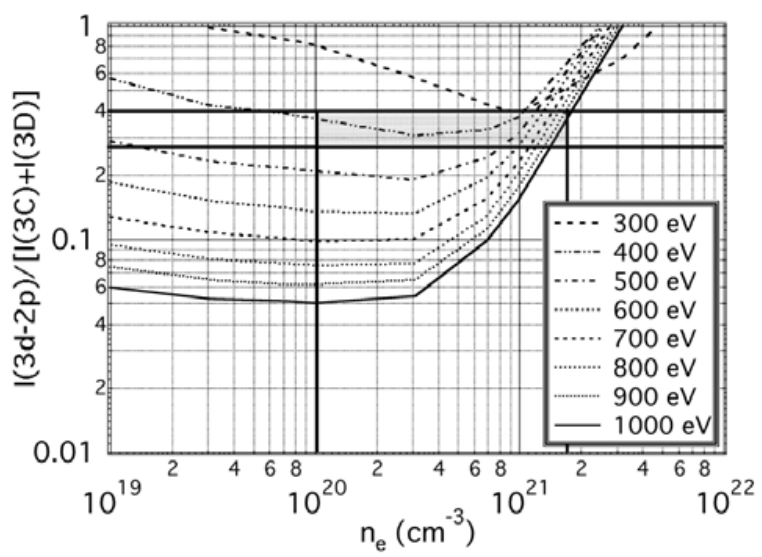

Figure 6. Rapport d'intensité des raies sodiumoïdes et néonoïdes calculé avec le code TRANSPEC, en fonction de la densité électronique et pour différentes températures électroniques. La region grisée indique les valeurs de densité et temperature déduites des rapports d'intensité des raies.

du $\mathrm{Sm}^{22+}$ au $\mathrm{Sm}^{30+}$. On retrouve certaines tendances déjà rencontrées dans le cas de l'émission de couche $\mathrm{K}$ : l'émission est plus intense en l'absence de la couche de V, et les structures spectrales tendent à disparaître lorsque cette dernière est présente. Ainsi, le «creux» juste avant 7,9 $\AA$, bien évident dans le cas du Sm pur, est pratiquement comblé dans le cas de la cible en V/Sm, probablement par un élargissement des structures spectrales voisines. Enfin, il convient de noter que le spectre émis par la cible de V/Sm semble insensible à une variation de durée laser. Ce résultat est encore en cours d'analyse.

\section{SIMULATIONS D'HYDRODYNAMIQUE RADIATIVE}

Le code d'hydrodynamique CHIVAS [11,12] a été modifié afin de traiter les irradiations extrêmes utilisées pour les expériences décrites (traitement des collisions électron-ion en «champ fort ») et ainsi fournir des indications sur les conditions du plasma émissif. La figure 8 montre les profils de la densité et de la température électroniques au pic de l'impulsion laser pour des cibles en $\mathrm{Al}$ et V/Al irradiées par une impulsion laser de $3 \mathrm{ps}$ de durée (pour des intensités égales à $2,7 \cdot 10^{18}$ et $2,3 \cdot 10^{18} \mathrm{~W} / \mathrm{cm}^{2}$ respectivement). Le profil temporel du laser utilisé dans le code tient compte du piédestal et reproduit donc fidelement le profil de l'impulsion laser utilisée dans l'expérience. Sur la figure, les traits pleins représentent le plasma d'aluminium et les traits pointillés celui de vanadium (lorsqu'il est présent). Il est intéressant d'observer que, dans le cas de la cible bicouche, seule la couche de vanadium s'expand 

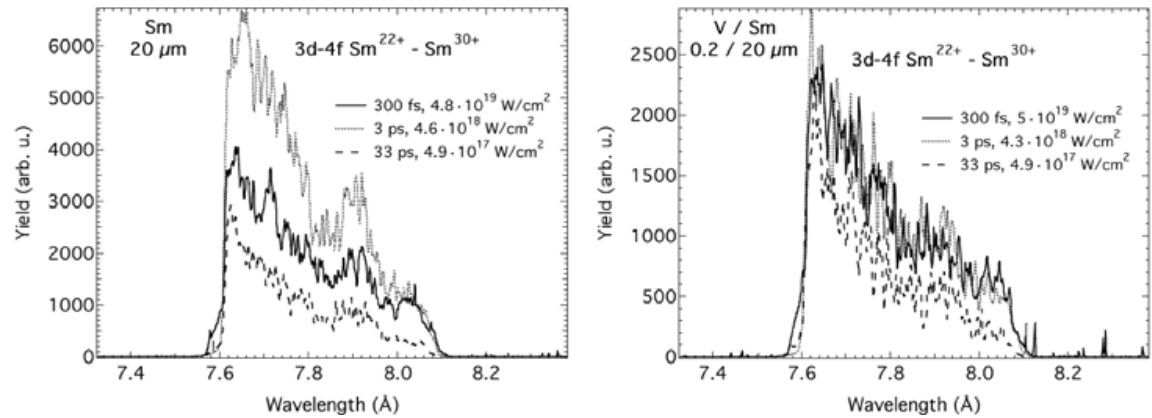

Figure 7. Spectres $3 d-4 f$ du Sm enregistrés pour trois conditions d'irradiation laser.
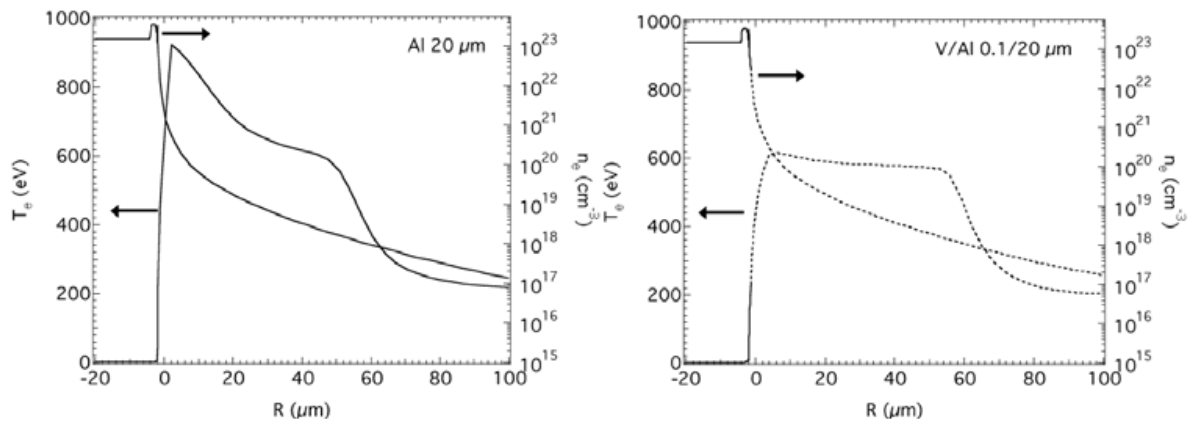

Figure 8. Profils de densité et température au pic de l'impulsion laser calculés avec le code CHIVAS, pour une cible en $\mathrm{Al}$ pur (à gauche) et pour une cible en V/Al (à droite). L'intensité laser utilisée dans la simulation était de $2.7 \cdot 10^{18} \mathrm{~W} / \mathrm{cm}^{2}$ et de $2.3 \cdot 10^{18} \mathrm{~W} / \mathrm{cm}^{2}$, respectivement.

dans le vide, l'aluminium restant pratiquement figé à sa position initiale. Il restera donc chaud et dense, et par conséquent émissif, plus longtemps, en accord avec les conclusions du paragraphe 3.1. Par contre, dans le cas de la cible d'Al pur, la température maximale atteinte est nettement plus élevée (de $300 \mathrm{eV}$ ) ce qui est cohérent avec les observations d'une raie He $\alpha$ plus intense.

\section{PREMIÈRES SIMULATIONS DE PHYSIQUE ATOMIQUE}

Les résultats du code CHIVAS présentés dans la section précédente (figure 8) ont été utilisés en entrée du code de cinétique atomique AVERROES/TRANSPEC [13,14]. La figure 9 montre les spectres ainsi calculés, au pic de l'impulsion laser, superposés aux spectres mesurés. Dans le cas de la cible en Al pur, bien que le rapport d'intensité entre la raie de résonance He $\alpha$ et les raies satellites lithiumoïdes soit relativement bien reproduit, TRANSPEC sous-estime très nettement les largeurs des raies. Ceci pourrait être dû à une sous-estimation de la densité électronique par le code d'hydrodynamique (et donc de l'élargissement collisionnel) provoquée soit par un traitement incorrect de l'absorption laser dans celuici (notamment des effets en champ fort) soit par une évaluation de l'intensité sur cible erronée. Toutefois, une explication plus probable pourrait être liée à la non prise en compte dans le code hydrodynamique de mécanismes d'interaction non linéaires (absorption anormale, ...) qui, pour des intensités supérieures à $10^{18} \mathrm{~W} / \mathrm{cm}^{2}$, génèrent une population non négligeable d'électrons supra-thermiques. Ceux-ci, en se propageant vers l'avant, créent dans la cible sur-critique des « trous » en couche interne, peuplant alors des niveaux doublement excités de nombre quantique élevé (dans le cas des expériences décrites dans ce papier, $1 s 2 \ln l^{\prime}$ par exemple). Leur dé-excitation induit une émission, dans le domaine $\mathrm{X}$, de raies satellites qui s'accumulent sur le flanc bleu/rouge de la raie de résonance associée et élargissent cette 

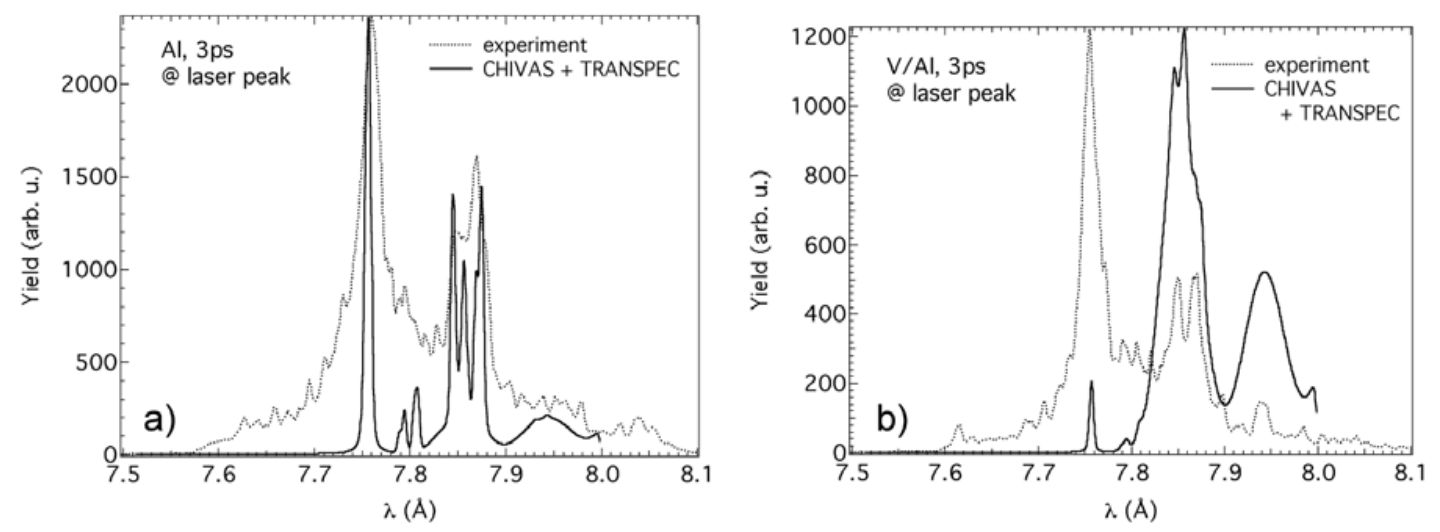

Figure 9. Spectres d'émission calculés en utilisant le code TRANSPEC comme post-processeur du code CHIVAS, comparés aux spectres mesurés. a) Cas d'une cible en $\mathrm{Al}$ pur, irradiée par une impulsion laser de 3 ps de durée, à $2.7 \cdot 10^{18} \mathrm{~W} / \mathrm{cm}^{2}$; b) cas d'une cible en V/Al, irradiée par une impulsion laser de 3 ps de durée, à $2.3 \cdot 10^{18} \mathrm{~W} / \mathrm{cm}^{2}$.

dernière. Dans le cas de la cible en V/Al, la situation est encore plus critique car même les intensités relatives des raies ne sont pas correctement simulées. L'existence d'une forte population d'électrons chauds, et donc d'un processus de chauffage additionnel ignoré dans les simulations, peut également être évoquée ; en effet, le spectre calculé correspond très clairement à une émission de plasma froid, pour laquelle les raies satellites dominent la raie de résonance.

\section{CONCLUSIONS}

En conclusion, nous avons mesuré les spectres d'émission de couche K, L et M de plasmas créés par des cibles de différents numéros atomiques et pour différentes conditions d'irradiation. Nous avons mis en évidence que, lorsque la durée de l'impulsion laser diminue (et donc l'intensité augmente), les structures spectrales tendent progressivement à s'estomper. Cette disparition peut être attribuée à un élargissement Stark important (lié à la forte densité du plasma émetteur) et à une accumulation de raies satellites d'ordre élevé (due à une forte populations d'électrons supra-thermiques). Pour interpreter plus finement les observations, l'effort déployé pour améliorer les simulations, aussi bien d'hydrodynamique radiative que de cinétique atomique, va être poursuivi. Les premiers calculs sont d'ores et déjà très prometteurs, car ils permettent de retrouver les principales caractéristiques observées dans le cas des cibles en Al pur, i.e., compte tenu de la forme temporelle de l'impulsion laser délivrée par l'installation 100TW, pour des plasmas émetteurs sous-denses.

Sur le plan expérimental, une prochaine campagne d'expérience sera menée sur l'installation 100TW. Dans cette campagne, nous projetons de doubler la frequence de l'impulsion laser, ce qui permettra de minimiser le piédestal dû à l'ASE et la génération d'électrons supra-thermiques. Il nous sera donc possible d'isoler l'influence de ces deux effets sur les spectres mesurés.

\section{Références}

[1] J.C. Gauthier et al., Phys. Plasmas 4, (1997) 1811

[2] K. Eidmann et al., J. Quant. Spectrosc. Radiat. Transfer 65, (2000) 173

[3] U. Andiel et al., Physz. Rev. E 63, (2001) 26407

[4] P. Audebert et al., J. Quant. Spectrosc. Radiat. Transfer 81, (2003) 19

[5] K. Eidmann et al., J. Quant. Spectrosc. Radiat. Transfer 81, (2003) 133

[6] S. Bastiani-Ceccotti et al., Appl. Phys. B 78, (2004) 905 
[7] D. Riley et al., J. Quant. Spectrosc. Radiat. Transfer 99, (2006) 537

[8] C. Chenais-Popovics et al., J. Phys. IV France 108, (2003) 137

[9] T. Hall, J. Phys. E: Sci. Instrum. 17, (1984) 110

[10] P. Gallant et al., Rev. Sci. Instrum. 71, (2000) 3627

[11] L. Bonnet et al., Inst. Phys. Conf. Ser. 140, (1995) 193

[12] S. Jacquemot et al., Advances of laser-matter interaction and inertial fusion (World Scientific Publishing Co., 1997)

[13] O. Peyrusse, J. Phys. B 33, (2000) 4303

[14] O. Peyrusse, J. Quant. Spectrosc. Radiat. Transfer 71, (2001) 571

[15] O. Peyrusse, Thèse de Doctorat 